\title{
Modeling of single pulse 3-D energy deposition profiles inside dielectrics upon fs laser irradiation with complex beam wavefronts J. Hoyo ${ }^{1}$, M. Galván-Sosa ${ }^{1}$, A. Ruiz de la Cruz ${ }^{1}$, E. J. Grace ${ }^{2}$, A. Ferrer ${ }^{3}$, J. Siegel ${ }^{1}$, J. Solis ${ }^{1}$ \\ ${ }^{1}$. Laser Processing Group; Instituto de Optica, CSIC, Madrid. España - 28006; ${ }^{2 .}$ Formerly at Photonics Group, Blackett Laboratory, Imperial College London, Prince Consort Road, London SW7 2BW, UK; ${ }^{3}$. Ultrafast Dynamics Group, Institute for Quantum Electronics, ETH Zurich, Wolfgang-Pauli-Strasse 16, 8093, Zurich, Switzerland
}

\begin{abstract}
Nonlinear laser processing of dielectrics with ultrafast lasers has been extensively studied over the last years and successfully applied to the production of photonics and micro-fluidic devices. Still, problems related to the presence of strong optical nonlinearities make it difficult to optimize the spatial intensity distribution in the focal region (SIDFR) in some cases. Methods providing a rapid estimate of the latter, even approximately, can be of great help for optimizing processing strategies and in other applications conditioned by nonlinear propagation like spatial soliton shaping. We have developed a numerical method for estimating the SIDFR inside a dielectric material, considering nonlinear absorption, nonlinear refraction and spherical aberration for laser beams with arbitrarily shaped wavefront. It is based on a generalized adaptive fast-Fourier evolver and has been successfully tested for flat wavefronts in subsurface processing. In this work we demonstrate its applicability to complex wavefronts, like those that can be generated with spatial light modulators (SLM). For this purpose the beam wavefront is described using Zernike polynomials before being propagated inside the material for different depths, pulse parameters. The results obtained show that under certain conditions, nonlinearities can be not only controlled and pre-compensated but also exploited for producing tailored SIDFRs.
\end{abstract}

Keywords: spatial shaping, fs-laser, complex wavefront, laser processing, nonlinear, dielectric, focal volume.

\section{INTRODUCTION}

The direct fs-laser ${ }^{1}$ writing technique (DWLT) has been extensively used in the fabrication of photonic and micro-fluidic devices ${ }^{2}$. This technique consists in focusing an ultrashort-pulsed laser beam inside a dielectric material while being translated with respect to the focus position. Due to photoionization and avalanche ionization processes, the energy of the laser is transferred to photogenrated free carriers. The excited carriers relax, transferring their energy to the lattice, which eventually undergoes a structural a modification. As these mechanisms are strongly dependent on the light intensity, the spatial distribution of the modification inside the material will depend on the intensity distribution ${ }^{3}$. Ideally, the production of functional structures implies controlling the SIDFR. However, the spatial intensity distribution will be affected by several factors, including non-linear propagation and aberrations ${ }^{4}$.

In this work, we use a simulation tool that allows calculating the propagation of a laser beam with an arbitrary wavefront using the nonlinear Schrödinger equation (NLSE) under simplified interaction conditions. With this software we can calculate the 3D SIDFR influenced by spherical aberration and nonlinear effects. We have used this tool to measure the nonlinear parameters of dielectrics, which usually are unknown except for a set of widely used and studied materials, and to pre-design the wavefront of the input beam in order to pre-compensate the distortion of the SIDFR upon nonlinear propagation and absorption. This tool can be similarly used for a broader aim, like studying laser-matter interaction when the beam wavefront cannot be described only by radius of curvature. 


\section{FORMALISM}

\subsection{Model}

Depending on the characteristics of a laser beam, its propagation inside a dielectric material will undergo effects that can range from diffraction, to nonlinear refraction, self-steepening or photoionization ${ }^{5}$. A detailed description of all these effects is beyond the aim of this manuscript.

In a general case, the propagation of a laser pulse inside a transparent material is given by the Maxwell and constitutive equations with additional correction terms associated to nonlinear polarization and free carrier density generation ${ }^{6}$. Assuming linear polarization and a slow varying envelope in the paraxial approximation, the evolution of the field adopts the form of the scalar $\mathrm{NLSE}^{7}$ :

$$
\begin{aligned}
& \frac{\partial A}{\partial z}=\frac{i}{2 k}\left(\frac{\partial^{2}}{\partial^{2} x^{2}}+\frac{\partial^{2}}{\partial^{2} y^{2}}\right) A+\frac{i k_{0} n_{2}|A|^{2}}{2 \eta_{0}} A-\frac{1}{2} \frac{W_{P I}(|A|) U_{i}}{|A|^{2}} A-i\left(\left.\frac{\partial^{2} k}{\partial \omega^{2}}\right|_{\omega_{0}}\right) \frac{\partial^{2} A}{\partial^{2} t^{2}}-\frac{\sigma}{2}\left(1+i \omega_{0} \tau_{c}\right) \rho A \\
& \text { ) }
\end{aligned}
$$

where the electric field is expressed as $\vec{E}(x, y, z, t)=\frac{1}{2} A(x, y, z, t) \exp \left[i\left(k z-\omega_{0} t\right)\right] \vec{e}_{x}+c c$, the refractive index and the intensity are defined as $n(I)=n_{0}+n_{2} I$ and $I=n_{0}|A|^{2} / 2 \eta_{0}, z$ is the propagation direction, $k_{0}$ and $\omega_{0}$ are the central wavenumber and frequency inside the material, $n_{2}$ the nonlinear refractive index of the material, $\eta_{0}$ is the vacuum impedance, $W_{P I}$ the photoionization coefficient, $U_{i}$ the material bandgap, $\rho$ the electron density (which is time-dependent) and $\sigma$ the cross section for inverse Bremstrahlung. The first term accounts for diffraction, the second one for nonlinear refraction, the third one for photoionization, the forth one for group velocity dispersion and the last one for plasma defocusing and absorption. Solving Eq. 1 requires considering also the temporal evolution of the free carrier density, considering the corresponding excitation and relaxation mechanisms ${ }^{8}$.

Solving the NLSE and rate equations often requires the use of numerical methods that normally involve large computational times (hours to days) ${ }^{9}$. However, there are situations where is not necessary to acquire an extremely detailed view of the process (i.e in terms of the temporal evolution of carrier density). This is the case, for example, when analyzing spatial soliton formation ${ }^{10}$, where the plasma effects can be neglected, or when trying to make an a priori first guess of optimized parameters for material laser processing using the DWLT ${ }^{11}$, where plasma effects can be, in a first crude approach, neglected. In these cases, we can use a simplified NLSE:

$$
2 i k \frac{\partial A}{\partial z}+\left(\frac{\partial^{2}}{\partial^{2} x^{2}}+\frac{\partial^{2}}{\partial^{2} y^{2}}\right) A+\frac{n_{2} k^{2}}{\eta_{0}}|A|^{2} A+i k \beta_{K}\left(\frac{n_{0}}{2 \eta_{0}}\right)^{K-1}|A|^{2 K-2} A=0
$$

where group velocity dispersion and plasma effects have been neglected, and the photoionization term has been replaced by an effective multiphoton absorption term involving the absorption of $K$ photons (defined as $\partial_{z} I=$ $-\beta_{K} I^{K}$ ), an approximation valid for weak fields (up to $\left.\sim 10^{13} \mathrm{~W} / \mathrm{cm}^{2}\right)^{6}$. It is worth noting that Eq. 2 in absence of nonlinear absorption corresponds to the Hemholtz equation for a Kerr medium under paraxial approximation ${ }^{12}$.

\subsection{Method}

In order to solve Eq. 2, a split-step numerical method is used. Neglecting time-dependent processes, calculation time is severely reduced as the beam propagation is calculated in quasi-stationary regime. This is equivalent to obtaining a snapshot of the SIDFR for the peak power of the beam, when the nonlinear effects are expected to be most intense. The code includes a library of optimized functions developed by E. Grace ${ }^{13}$ that contain routines to dynamically adjust the step size, the spatial dimensions (perpendicular to propagation direction) and resolution of the mesh that represent the field in order to avoid unnecessary calculations and further reduce the calculation time. This allows obtaining results in short times (seconds to several minutes) even in an average laptop computer. The efficiency of the calculation process allows thus testing a large number of processing conditions in an affordable time.

The code is divided into three main stages, represented in Fig. 1. The first one starts defining the initial field as an amplitude and wavefront: $A(x, y)=|A(x, y)| \exp [i k \Delta(x, y)]$. The intensity is defined as an elliptic Gaussian 
$|A(x, y)|=A_{0} \exp \left(-\frac{x^{2}}{R_{x}^{2}}\right) X \exp \left(-y^{2} / R_{y}^{2}\right) \exp \left(-t^{2} / R_{t}^{2}\right)$ for $t=0$, when beam has maximum intensity. Special beam intensity distributions like those corresponding "slit-shaped beams"14 are not directly implemented because the code is essentially based in the use of Fast Fourier Transforms (see below) and this kind of profiles requires a huge number amount of points to represent properly the electric field distribution. Slit shaped beams are modeled as elliptical Gaussian beams with a given aspect ratio leading to the same peak intensity. These kind of beams can produce circular SIDFRs, which are very useful for waveguide writing using the DWLT.

The wavefront of the beam in the code is usually defined in a base of Zernike polynomials $\Delta(r, \varphi)=$ $\sum_{n} A_{n} Z_{n}(r, \varphi)$, being $r=\sqrt{x^{2}+r^{2}} / R_{r e f}, R_{\text {ref }}$ the reference radius the Zernike polynomials are referred to, and $\varphi=\operatorname{asin}(y / x)$. This is particularly useful for simulating wavefronts generated by Spatial Light Modulators (SLM), although arbitrary discretized wavefronts can be also propagated with a larger computational cost. We chose this particular combination where the field is described in Cartesian coordinates and the wavefront in Zernike polynomials (described in polar coordinates) because the Cartesian coordinates are adequate to model a real SLM made with pixels and the polar coordinates suits a circular beam.

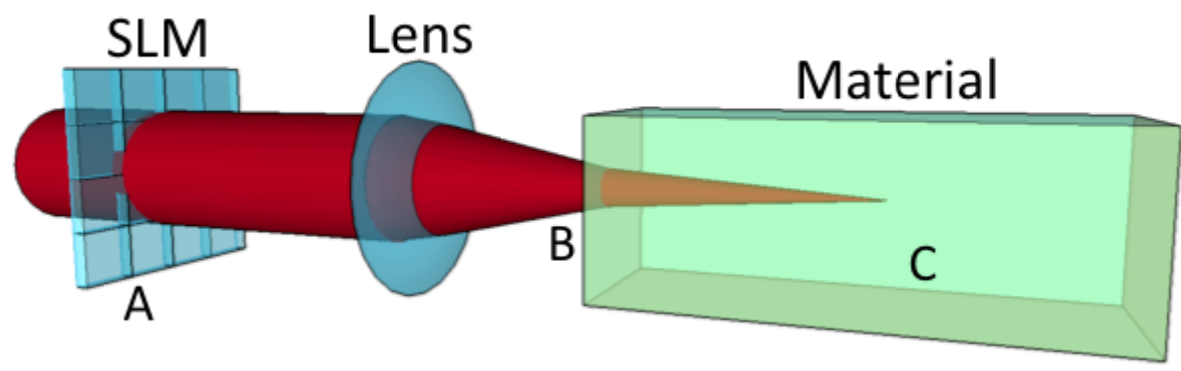

Figure 1. Schematic representation of the simulated experiment. The beam wavefront is defined at A. The beam is then propagated linearly through the lens until B, where interface effects are calculated. Finally, it is propagated inside the material (C).

In the second stage, the initial beam travels in air to be focused by a lens through a strictly linear medium at the interface with the dielectric. This propagation part is calculated using the Fresnel equation, but not in its original expression (computationally highly costly) but dividing it into several simpler operations ${ }^{15}$ like scaling, introduction of a quadratic phase, or a Fourier transform. Then, the code introduces the effects derived from a change in the refractive index: reflection, refraction, a change in the electric field amplitude and spherical aberration (SA). SA is computed adding a new wavefront to the field ${ }^{16}$ :

$$
\Delta_{S A}(\rho)=d N A\left(\sqrt{\operatorname{cosec}^{2} \beta-\rho^{2}}-\sqrt{\operatorname{cosec}^{2} \alpha-\rho^{2}}\right)
$$

where $d$ is the focusing depth inside the dielectric, $N A=n_{0} \sin (\alpha)$ is the numerical aperture, $n_{0}$ inc $\sin (\alpha)=$ $n_{0 d i} \sin (\beta)$ corresponds to the Snell law and $\rho=\sin (\alpha) / \sin (\phi)$ being $\phi$ the incident angle. This is more accurate than just using the first order spherical aberration $Z_{8}(\rho)=6 \rho^{4}-6 \rho^{2}+1$.

When the beam enters the dielectric material, the third stage of the code takes over. First of all, the code tests if the field is aliased in the real or Fourier spaces by calculating the fraction of energy in the outer part of the field mesh. If the fraction of energy is above a certain tolerance, the field is declared aliased and the simulation has to be re-started from the beginning changing the initial beam representation parameters until aliasing is suppressed

If the field is free of aliasing, the propagation of the field inside the dielectric using Eq. 2 begins. The propagation is divided into small $\Delta z$ steps in the propagation direction in an iterative way, calculating $A(z+\Delta z)$ from $A(z)$. For each step several calculations are made. First, the linear propagation is calculated over $\Delta z / 2$ in the Fourier space, followed by the nonlinear propagation over $\Delta z$ both in real and Fourier spaces, and finalized with the linear 
propagation over $\Delta z / 2$ again in Fourier space. This can be seen as applying the nonlinear propagation to an averaged field over linear propagation.

Before proceeding to the next step, two tests are made. First of all, if $z>0$ it calculates the propagation from $A(z$ $\Delta z)$ to $A^{\prime}(z+\Delta z)$ with a step of $2 \Delta z$ and measures the distance between $A$ and $A^{\prime}$. If the distance is higher than a certain tolerance, the step distance $\Delta z$ is reduced and the step propagation is recalculated. On the other hand, if it is smaller, the propagation is declared valid and the code will proceed to the next step. If it is much smaller, the code increases $\Delta z$ for the next step to increase calculation time. The second test calculates the fraction of energy in the outer part of the mesh both in real and Fourier spaces searching for aliasing effects. If the fraction is very low it reduces the space boundaries in that space (reducing the resolution) and calculates the field in the conjugate space to avoid oversampling. If the field is aliased in one dimension, the code increases the resolution in the non-aliased space and calculates the field in the conjugate space accordingly. If the required number of points is too high or the field is aliased in both dimensions, it reduces $\Delta z$ and recalculates the step.

Repeating this procedure the code calculates the whole propagation until it reaches the desired representation range. However, it can happen that the code requires a step size $\Delta z$ too low for the method to be efficient. In that case, the calculation is aborted and must be restarted from the beginning. In most cases, varying the initial simulation parameters of the beam will lead to a more favorable situation so the calculation can be completed. However, there will be cases where this is not possible, for example, when the focusing depth and numerical aperture are really elevated, or the nonlinearities are extreme. But in this case, the approximations performed to obtain Eq. 4 won't be valid anymore and a more general method would be required anyway.

\section{RESULTS}

\subsection{Precompensation of nonlinearities}

The first row of Fig. 2 shows the SIDFR of the beam for different energies with a flat wavefront, including SA effects at the interface. The used parameters are similar to a typical DWLT experiment in a phosphate glass: beam radii $R_{x}=3.7 \mathrm{~mm}$ and $R_{y}=R_{x} / 20$, pulse duration (FWHM) $120 f \mathrm{~s}$, wavelength $800 \mathrm{~nm}$, focal length $20 \mathrm{~mm}$, focusing depth $0.5 \mathrm{~mm} \mathrm{n} n_{0}=1.56, \mathrm{n}_{2}=1.0 \times 10^{-20} \mathrm{~m}^{2} / W$ and $\beta_{3}=2.0 \times 10^{-30} \mathrm{~m}^{3} / \mathrm{W}^{2}$. Fig. 2a (lowest energy) presents the typical circular disc shape of a slit shaped beam ${ }^{17}$, only slightly distorted due to SA. This SA distortion can be precompensated ${ }^{16}$ adding an extra wavefront, but that is beyond the aim of this manuscript. It can be seen that as the energy increases (Fig. 2a-d), the SIDFR gets distorted more and more, acquiring s triangular shape, increasing its size and showing prefocal energy depletion ${ }^{18}$, energy absorption before the beam reaches the linear focal region.

In order to determine the feasibility of pre-compensating nonlinear and spherical aberration effects, we have used a non-flat wavefront given by only one Zernike polynomial to show the versatility of the technique. We have chosen the first order spherical aberration polynomial, $Z_{8}$, with negative amplitude and a reference radius equal to the beam radius because this aberration tends to elongate the focal volume deeper inside the material, as opposite to non-linear refraction.
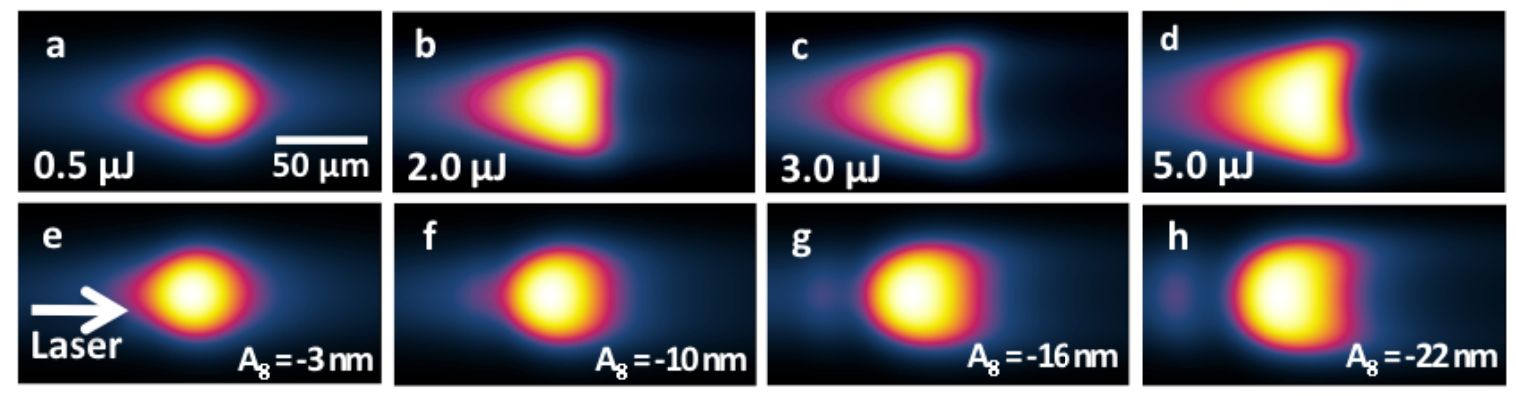

Figure 2. Calculated focal intensity distribution cross-sections of 200x100 $\mu \mathrm{m}$ along the propagation direction for a slit shaped beam for different pulse energies. The first row shows the profile induced by beams with an initially flat wavefront, including SA effects at the interface over nonlinear absorption and nonlinear propagation. The second correspond to the simulated effect of modifying the wavefront with a SLM introducing an additional 
spatial phase given by the first order spherical aberration Zernike polynomial with different amplitudes, shown in figure, and the same reference radius of $3.7 \mathrm{~mm}$. Each image is normalized independently.

The second row of Fig. 2 shows the pre-compensated SIDFR for the same energies. The amplitude coefficient value has been chosen to maintain the circular shape of the SIDFR and its Rayleigh range. Below $3 \mu J$, the SIDFR recovers the circular disk shape. For higher energies, the wavefront deformation introduced is unable of pre-compensating the whole nonlinear distortion and the SIDFR's show a flat edge in its deeper side. This result may be improved using more complex wavefronts that involve higher order Zernike polynomials, and an optimization algorithm. However, the result obtained by using just one polynomial for linear pre-compensation of nonlinear propagation effects can be considered as excellent.

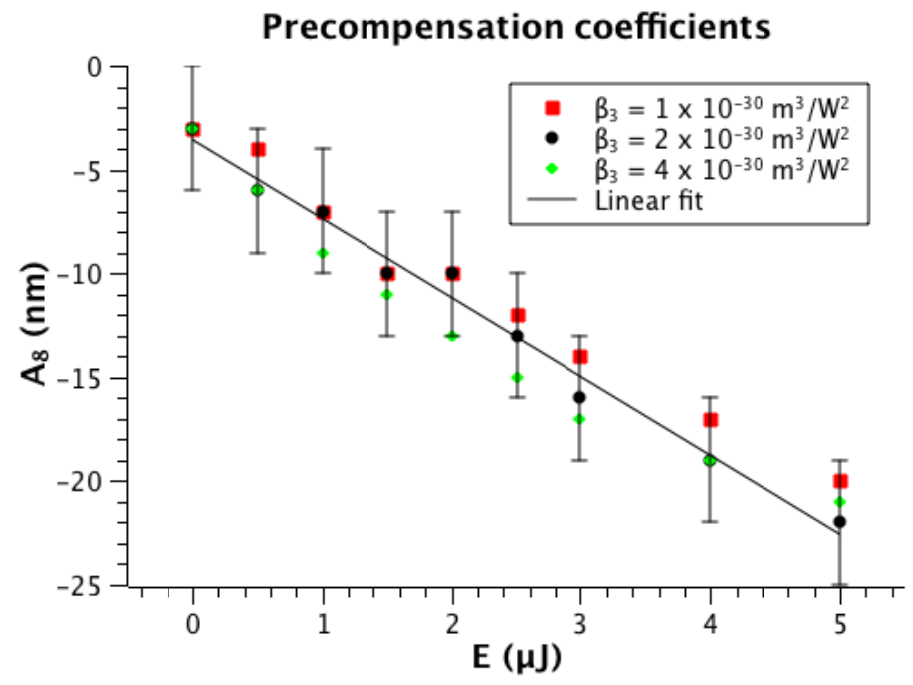

Figure 3. Pre-compensating amplitude coefficients for different three-photon absorption coefficients. Error bars are only shown for $\beta_{3}=2 \times 10^{-30} \mathrm{~m}^{3} / W^{2}$ for clearness.

The amplitude values used for pre-compensation as a function of the pulse energy can be seen in Fig 3 . The error bars show the uncertainty in the coefficient determined for a resolution of $1 \mu \mathrm{m}$ in the spatial variables (the resolution changes during calculation, but cannot exceed this maximum resolution). This uncertainty is calculated by the minimum amount we have to increase the amplitude of the Zernike polynomial in order to measure a change in the Rayleigh range equal to the spatial resolution in the simulation. Although apparently high, the uncertainty can be reduced increasing the spatial resolution at the cost of increasing the calculation time. In the case of Fig. 3, the resolution was chosen such that the calculation time remained below 3 minutes in an ordinary laptop computer, in order to show the versatility of the method. It can be seen that the $A_{8}$ scales nearly linearly with pulse energy, leading to a linear fit, $A_{8}=a+b E$ where $a=-3.5 \pm 1.7 \mathrm{~nm}$ and $b=-3.81 \pm 0.065 \mathrm{~nm} / \mu \mathrm{J}$.

Fig 3 also shows the influence of the material parameters in the pre-compensation coefficients. It shows the amplitude values required for several different three-photon absorption coefficients corresponding to the double and the half of the one of the initial simulations. It can be seen that all the pre-compensation amplitudes coincide (within the indicated uncertainty interval) with initially determined ones. We can thus conclude that the influence of the material parameters on the pre-compensation amplitude is not abrupt, facilitating the initial search.

\subsection{Wavefront design}

In this section we show how the use of non-flat wavefronts may lead to exotic SIDFRs and that these tailored wavefronts can be used to exploit material nonlinearities. An illustrative example is shown in Fig.4a. In this case, we have used the same laser and material parameters, a non-slit-shaped circular beam of $2.5 \mathrm{~mm}$ radius, $0.5 \mu \mathrm{J}$ pulse energy, and a non-flat wavefront given by $Z_{14}(\rho)=\left(10 \rho^{4}-12 \rho^{2}+3\right) \rho \sin \varphi$, with an amplitude of 1 $\mu \mathrm{m}$ and a reference radius of $12.5 \mathrm{~mm}$, focused at a depth of $0.3 \mathrm{~mm}$ using the same focal length of $25 \mathrm{~mm}$ (NA of 0.10 in this case). 
For these conditions, when the beam approaches the focus position (Fig 4a), the maximum of energy is concentrated along two high intensity spots separated about $15 \mu \mathrm{m}$. When the pulse reaches the focal position (Fig $4 \mathrm{~b}$ ), it is divided into four different high intensity spots forming a rhomb with "hot spots" separated about 10 $\mu m$. Finally, when the pulse is beyond the focal position (Fig 4c) is again divided into two high intensity spots, like before reaching the focus, but oriented perpendicularly.

Integrating the intensity along $0.4 \mathrm{~mm}$ of the propagation direction (Fig. 4d) with the focal position in the middle, it can be seen that the region of highest intensity forms a square, with a weakly irradiated center. This image illustrates the spatial distribution of intensity experienced by the material if it is moved along the beam. Irradiating a glass that undergoes a refractive index diminution upon irradiation, and moving the sample along the propagation direction of the beam, a depressed index waveguide ${ }^{2}$ might be produced at once using only one single scan. The overall dimension of the waveguide could be controlled by changing $N A, A_{14}$, or adding more polynomials to the wavefront.
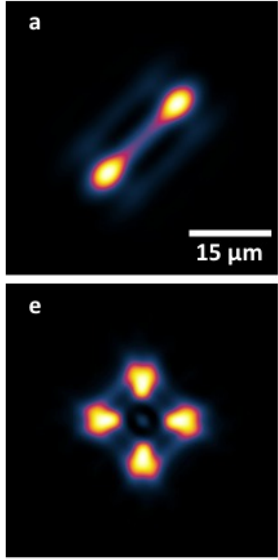
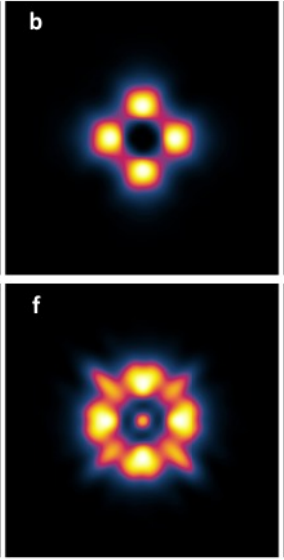
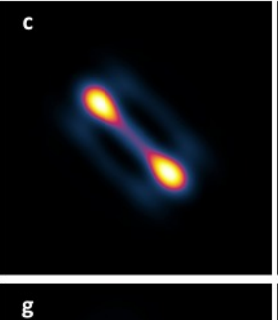

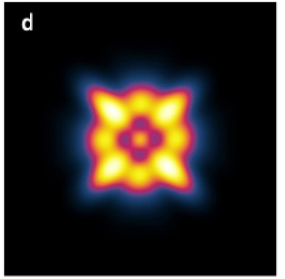

g

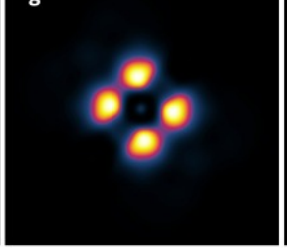

h

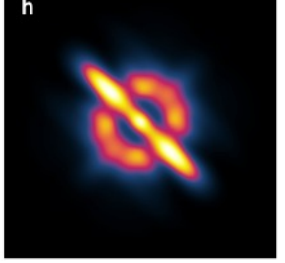

Figure 4. Calculated intensity cross-section distributions of $50 \times 50 \mu \mathrm{m}$ perpendicular to the propagation direction at a depth of (a) $0.2 \mathrm{~mm}$, (b) $0.3 \mathrm{~mm}$ and (c) $0.4 \mathrm{~mm}$ for a beam of $0.5 \mu \mathrm{J}$ focused $0.3 \mathrm{~mm}$ below the surface. (d) is the integration of intensity along $0.4 \mathrm{~mm}$ of propagation centred in the focus, and reflects the effect of irradiating the material while moving it along the focal intensity distribution. (e) is the transversal intensity profile of the same beam focused $3.0 \mathrm{~mm}$ below the surface and (f) the integration along propagation, analogous to (b) and (d) respectively. ( $g$ ) is the intensity of a beam of $3 \mu \mathrm{J}$ focused at $0.3 \mathrm{~mm}$ of depth, and (h) the integration along propagation. All images are shown in linear scale and normalized to their maximum value.

If we focus the same beam ten times deeper it can be seen that the focal cross section intensity distribution at the focus (Fig 4e) and the integration over de $0.4 \mathrm{~mm}$ of propagation (Fig 4f) are even better for the waveguide irradiation purpose, as the intensity is mainly concentrated in the edges and less in the corners and the center (Fig 4f), allowing the production of a long waveguide. If we increase the energy of the beam to $3.0 \mu \mathrm{J}$, a small distortion of the focal intensity cross-section can be appreciated (Fig 4g). However, the integrated image (Fig 4h) shows that for high energies, the parameters of the wavefront or/and the $N A$ should be adjusted in order to achieve the desired modification.

\section{CONCLUSION}

We have shown a new method to expand the capabilities of the direct writing laser technique. Using our model we can simulate the nonlinear propagation of arbitrary shaped wavefront laser beams inside dielectric materials. This can be used to design wavefronts capable of pre-compensating spherical aberration and nonlinear propagation-related deformations of the focal intensity distribution. Its use may open a route for tailoring intensity distributions taking advantage of material nonlinearities. 


\section{ACKNOWLEDGEMENTS}

This work was partially supported by the Spanish Ministry of Economy and Competitiveness under TEC201122422. J. H. acknowledges funding from the JAE CSIC Program (pre-doctoral fellowships, co-funded by the European Social Fund).

\section{REFERENCES}

[1] K.M. Davis, K. Miura, N. Sugimoto, and K. Hirao, "Writing waveguides in glass with a femtosecond laser", Opt. Lett. 21, (1996) 1729-31.

[2] Osellame R, Cerullo G, Ramponi R. Ed. "Femtosecond Laser Micromachining: Photonic and Microfluidic Devices in Transparent Materials", New York: Springer; 2012. ISBN 978-3-642-23366-1

[3] A. Ferrer, A. Ruiz de La Cruz, D. Puerto, W. Gawelda, Vallés J. A., M.A. Rebolledo, V. Berdejo, J. Siegel, J. Solis, and A.R. de La Cruz, "In situ assessment and minimization of nonlinear propagation effects for femtosecond-laser waveguide writing in dielectrics", JOSA B 27 (2010) 1688-1692.

[4] C. Hnatovsky, R.S. Taylor, E. Simova, V.R.V. Bhardwaj, D.M.D. Rayner, and P.B. Corkum, "Highresolution study of photoinduced modification in fused silica produced by a tightly focused femtosecond laser beam in the presence of aberrations", Jour. of App. Phys. 98013517 (2005).

[5] A. Couairon and a. Mysyrowicz, "Femtosecond filamentation in transparent media," Phys. Rep. 441, 47-189 (2007).

[6] L. Sudrie, A. Couairon, M. Franco, B. Lamouroux, B. Prade, S. Tzortzakis, and A. Mysyrowicz, "Femtosecond laser-induced damage and filamentary propagation in fused silica," Phys. Rev. Lett. 89, 186601 (2002).

[7] Y. Liu, Y. Brelet, Z. He, L. Yu, S. Mitryukovskiy, A. Houard, B. Forestier, A. Couairon, and A. Mysyrowicz, "Ciliary White Light: Optical Aspect of Ultrashort Laser Ablation on Transparent Dielectrics," Phys. Rev. Lett. 110, 097601 (2013).

[8] G. I. Stegeman, "Optical Spatial Solitons and Their Interactions: Universality and Diversity," Science 286, $1518-1523$ (1999).

[9] B. Rethfeld, "Unified Model for the Free-Electron Avalanche in Laser-Irradiated Dielectrics," Phys. Rev. Lett., 92, 187401 (2004).

[10] S. W. Winkler, I. M. M. Burakov, R. Stoian, N. M. M. Bulgakova, A. Husakou, A. Mermillod-Blondin, A. Rosenfeld, D. Ashkenasi, and I. V. V. Hertel, "Transient response of dielectric materials exposed to ultrafast laser radiation," Appl. Phys. A 84, 413-422 (2006).

[11] A. Ferrer, A. R. de La Cruz, D. Puerto, W. Gawelda, Vallés J. A., M. Á. Rebolledo, V. Berdejo, J. Siegel, and J. Solis, "In situ assessment and minimization of nonlinear propagation effects for femtosecond-laser waveguide writing in dielectrics," JOSA B, 27,1688-1692 (2010).

[12] G. Fibich and B. Ilan, "Self-focusing of elliptic beams: an example of the failure of the aberrationless approxi-mation," J. Opt. Soc. Am. B 17, 1749 (2000).

[13] E. J. Grace, "GAFFE: A toolbox for solving evolutionary nonlinear PDEs", (2009). (Available from ww.mathworks.co.uk/matlabcentral/fileexchange/24016).

[14] Y. Cheng, K. Sugioka, K. Midorikawa, M. Masuda, K. Toyoda, M. Kawachi, and K. Shihoyama, "Control of the cross-sectional shape of a hollow microchannel embedded in photostructurable glass by use of a femtosecond laser.," Opt. Lett. 28, 55-7 (2003).

[15] J. Goodman, "Introduction to Fourier optics", Mc Graw Hill, New York, 1996.

[16] M. J. Booth, M. A. Neil, T. Wilson, "Aberration correction for confocal imaging in refractive index mismatched media", Jour. of Micr. 192(2) (1998) 90-98. 
[17] M. Ams, G. Marshall, D. Spence M. J. Withford, "Slit beam shaping method for femtosecond laser directwrite fabrication of symmetric waveguides in bulk glasses", Opt. Express 13 (2005) 5676-81.

[18] D. Rayner, A. Naumov, P.B. Corkum, "Ultrashort pulse non-linear absorption in transparent media", Opt. Express 13 (2005) 3208-17. 